\title{
CONSÉQUENCES INDUSTRIELLES ET ÉCOLOGIQUES DE L'INTRODUCTION DE NOUVELLES ESPĖCES DANS LES HYDROSYSTEMMES CONTINENTAUX : LA MOULE ZÉBRÉE ET AUTRES ESPĖCES INVASIVES.
}

\author{
M. KHALANSKI \\ EDF DER Département Environnement, 6 quai Watier, 78401 Chatou Cedex, France.
}

\section{RÉSUMÉ}

Les eaux de surface sont prélevées pour divers usages industriels, parmi lesquels la production d'énergie électrique représente une large part. Dans les circuits d'eau brute se fixent de nombreuses espèces d'organismes aquatiques, qui perturbent le fonctionnement des installations et sont susceptibles d'entraîner l'indisponibilité de certaines d'entre elles.

Dans certains cas, l'irruption de nouvelles espèces pose un problème grave à l'industrie. L'introduction de deux espèces de moules zébrées (genre Dreissena) dans les Grands Lacs américains, puis leur extension progressive vers le sud des États-Unis au cours des dernières années, se chiffre par un dommage pour l'industrie estimé à cinq milliards de dollars par an en 2000. L'impact écologique de la moule zébrée en Amérique du Nord a fait l'objet de nombreux travaux; dans la phase d'extension rapide de l'espèce, il semble significatif.

En Europe, la moule zébrée constitue aussi une menace pour les circuits industriels; c'est pourquoi cette espèce a fait l'objet de nombreuses études, notamment en France sur la Seine, le Rhône et la Moselle. Deux nouvelles espèces invasives sont récemment apparues dans les cours d'eau européens : il s'agit du clam asiatique Corbicula fluminea et d'un amphipode : Corophium curvispinum. Elles s'ajoutent à la liste faunistique des organismes observés dans les circuits de centrales thermiques installées sur les cours d'eau français : spongiaires, hydraires, bryozoaires, mollusques.

Pour assurer la disponibilité des circuits, il faut pouvoir contrôler le développement de ces espèces; ce qui implique une connaissance approfondie de leur biologie et de leur écologie. Les méthodes de lutte actuellement mises en oeuvre au plan industriel, ou qui sont à l'étude, sont brièvement passées en revue.

Mots-clés : espèces invasives, hydrosystèmes continentaux, moule zébrée, salissures biologiques, conséquences industrielles, impact écologique.

\section{INDUSTRIAL AND ECOLOGICAL CONSEQUENCES OF THE INTRODUCTION OF NEW SPECIES IN CONTINENTAL AQUATIC ECOSYSTEMS : THE ZEBRA MUSSEL AND OTHER INVASIVE SPECIES.}

\begin{abstract}
Surface water is withdrawn from rivers for various industrial uses; among these, power production accounts for a large proportion. Many aquatic species settle in the raw water circuits of power plants, disrupting their operation and occasionally putting the whole plants out of use.
\end{abstract}


In certain cases, the arrival of new species raises a serious problem for the industry. The introduction of two Dreissena species in the American Great Lakes, followed by their gradual spread southwards in recent years, has generated industrial damage estimated at five billion US $\$$ per year by the turn of the century. The ecological impact of the zebra mussel in North America has been widely studied and is thought to be considerable during the phase of rapid spreading of the species.

In Europe as well, the zebra mussel constitutes a threat for industrial circuits. For this reason, the species has been the object of numerous studies, particularly in France in the Seine, Rhône and Moselle rivers. Two new invasive species have recently appeared in European rivers : the Asian clam Corbicula fluminea and an amphipod: Corophium curvispinum, thus adding to the list of organisms (sponges, hydroids, bryozoans, molluscs) previously found in power plant circuits on French rivers.

To ensure the availability of plant circuits, it is essential to be able to control the development of these species. This, in turn, implies in-depth knowledge of them in both biological and ecological terms. This paper presents a brief overview of present industrial methods, or methods now under study, for checking the spread of such organisms.

Key-words : invasive species, inland waters, zebra mussel, biofouling, industrial consequences, ecological impact.

\section{LE CONTRÔLE DES SALISSURES BIOLOGIQUES : UNE NÉCESSITÉ TECHNIQUE ET ÉCONOMIQUE}

De nombreuses installations prélèvent des eaux de surface pour des usages directs (production d'eau et de produits alimentaires élaborés, irrigation) ou indirects, essentiellement pour assurer la réfrigération des machines. Le fonctionnement des centrales thermiques dépend étroitement de la disponibilité des circuits de réfrigération alimentés en eau brute. Sur le plan économique, l'élimination des causes de dysfonctionnement des circuits de réfrigération représente un enjeu important.

Le colmatage des filtres et des échangeurs thermiques par des organismes aquatiques a été depuis longtemps identifié par les exploitants comme un risque majeur contre lequel il faut se prémunir. Le colmatage peut se produire par l'aspiration aux prises d'eau de grandes quantités d'organismes végétaux ou animaux, ou par le développement excessif d'organismes fixés sur les parois des circuits. Ces " salissures biologiques " se détachent et l'entraînement de parties dures (coquilles...) dans les échangeurs peut les rendre indisponibles. Les coquillages fixés ou déposés dans les conduites produisent en outre des quantités importantes de vase organique, constituée par leurs déjections : fèces et pseudo-fèces, qui se déposent dans les échangeurs thermiques et augmentent la fréquence des lavages.

C'est pourquoi les producteurs d'électricité, et plus généralement les utilisateurs d'eaux douces ou marines, se préoccupent de protéger leurs circuits contre le développement des salissures biologiques. Ainsi, depuis le début des années 1970, des études sont effectuées à EDF, afin de mieux connaître la biologie des principales espèces de salissures et de définir les moyens permettant de les éliminer si besoin.

Dans ce mémoire, nous tenterons d'illustrer l'ampleur du problème à partir d'un exemple particulièrement démonstratif : l'invasion de l'Amérique du Nord par la moule zébrée, les dommages causés aux industries et l'impact écologique qui en résulte. Cet événement marquant pour les hydrosystèmes nord-américains a suscité une masse considérable d'études et de recherches sur la biologie et l'écologie de ce bivalve, comme sur les méthodes de contrôle dans les circuits industriels.

Nous présenterons ensuite, succinctement, les principales salissures biologiques présentes dans les circuits industriels en France à la fin de l'année 1995 et les espèces invasives exotiques récemment introduites dans les eaux continentales françaises. Les moyens mis en oeuvre ou à l'étude pour contrôler les salissures biologiques dans les centrales thermiques seront enfin abordés. 


\section{BRÈVE RELATION de L'INVASION DE L'AMÉRIQUe DU NORD PAR LA MOULE ZÉBRÉE}

2.1. L'installation de la moule zébrée en Europe Occidentale et en Amérique du Nord

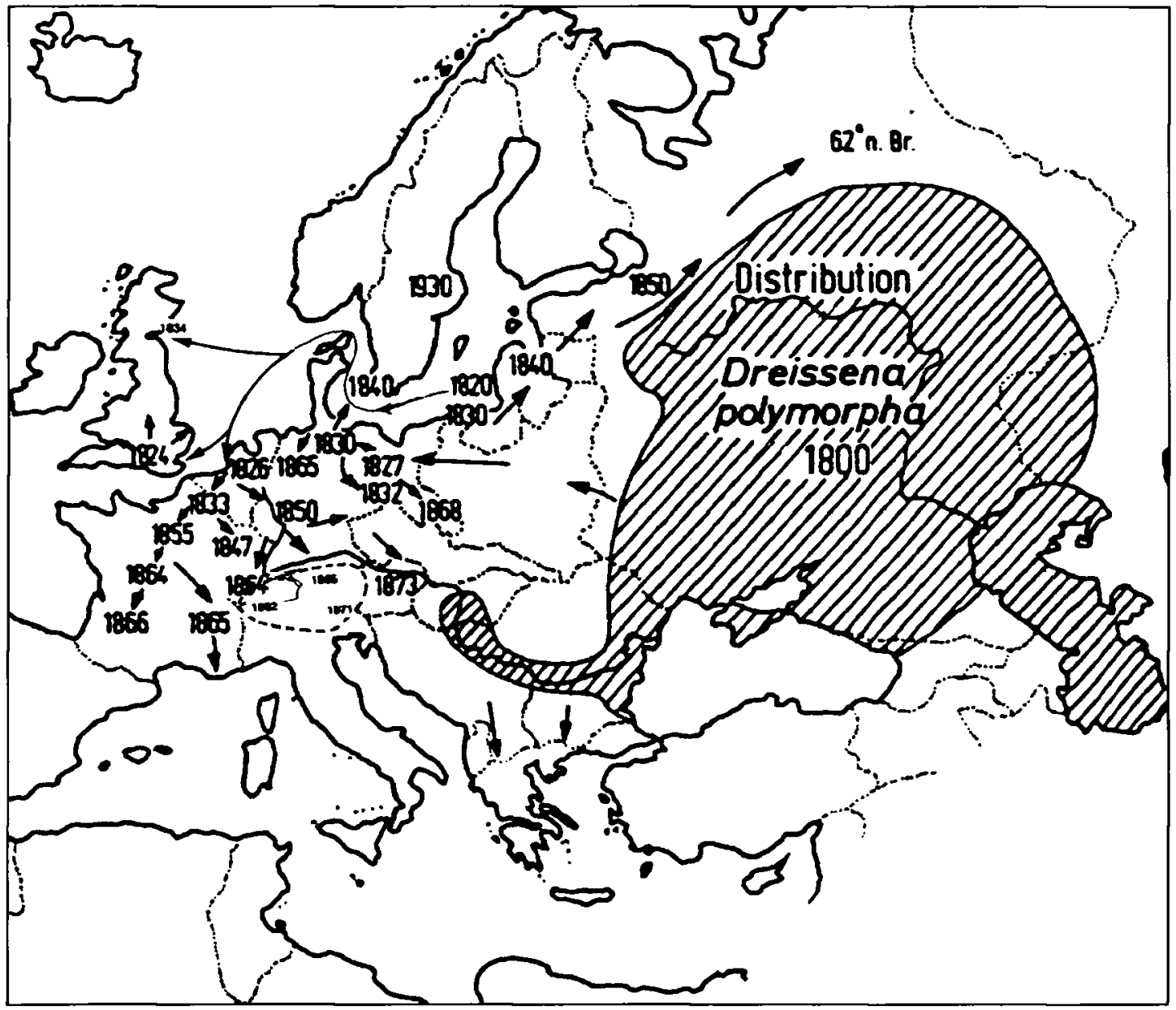

Figure 1

Extension de $D$. polymorpha en Europe Occidentale, au 19ème siècle. D'après MORTON, 1993 et KINZELBACH, 1992.

Figure 1

Spread of D. polymorpha in Western Europe, during the XIXth century. After MORTON, 1993 and KINZELBACH, 1992.

Originaire de la région de la Mer Noire et de la Caspienne, la moule zébrée, Dreissena polymorpha Pallas, a progressivement colonisé les rivières européennes. Au siècle dernier, sa progression vers l'ouest (schématisée sur la figure 1) a été favorisée par le développement des canaux au début de l'ère industrielle (MORTON, 1993; KINZELBACH, 1992). Elle a atteint, en quelques décennies, la France et les îles britanniques. La dreissène est aujourd'hui présente dans tous les grands bassins hydrographiques français, où elle a pris une importance plus ou moins grande (LÉGLIZE et OLLIVIER, 1981; TESTARD, 1990). Elle présente ainsi des populations très denses en Moselle et dans une partie du bassin de la Seine, son développement semble moindre dans le Rhône et elle est peu abondante dans les bassins de la Loire et de la Garonne. 
En Amérique du Nord, la première observation de moules zébrées a été rapportée en juin 1988 dans le lac Saint-Clair, un plan d'eau situé entre le lac Erié et le lac Huron. En fait, les experts estiment actuellement que l'introduction de l'espèce date vraisemblablement de 19851986 (SEA GRANT ZEBRA MUSSEL REPORT, 1995). Elle se serait faite à partir de larves émises par des moules européennes transportées avec l'eau douce de ballast des bateaux de haute mer. Ceux-ci remontent en effet le Saint-Laurent depuis 1959, pour atteindre les métropoles industrielles installées au bord des Grands Lacs.

L'identité de l'envahisseur semblait clairement établie au début de l'infestation. Toutefois, certaines populations présentes dans les Grands Lacs se distinguaient par la forme de leur coquille. Actuellement, deux espèces ont été identifiées : Dreissena polymorpha Pallas, la plus répandue, et la " quagga mussel ", identifiée comme étant Dreissena bugensis Andrusov, 1897 (SPIDLE et al., 1994).

Dès 1989, les premières nuisances se sont manifestées par le colmatage des prises d'eau d'usines de production d'eau alimentaire. A partir de 1990, tous les circuits d'eau brute ont été concernés, qu'il s'agisse de bateaux ou d'installations à terre. La moule zébrée a trouvé dans les Grands Lacs américains les conditions favorables à sa croissance et à sa reproduction.

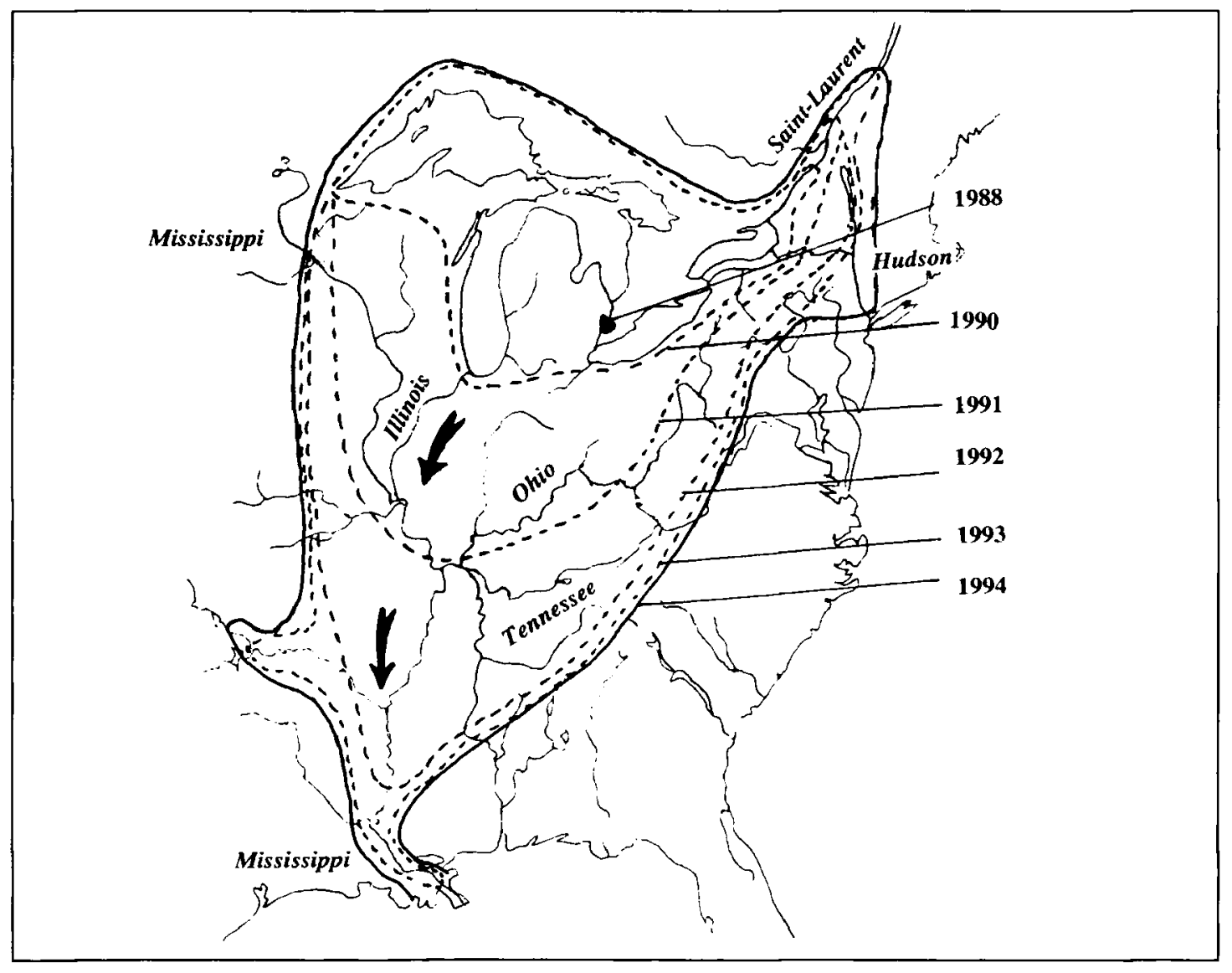

Figure 2

Distribution de $D$. polymorpha en Amérique du Nord, de 1988 à fin 1994. D'après CLAUDI et MACKIE, 1994, et les cartes éditées par the US Department of Interior à partir de la base de données du National Biological Service " Non Indigenous Aquatic Species Data Base ".

Figure 2

Distribution of D. polymorpha in North America, from 1988 to the end of 1994. After CLAUDI and MACKIE, 1994, and maps of the US Department of Interior. 
Comme la moule marine, elle possède des larves planctoniques qui assurent sa rapide dissémination en milieu aquatique et un appareil de fixation constitué de filaments adhérant aux substrats durs : le byssus. Elle peut couvrir toutes les surfaces disponibles, se fixer sur les coquilles de ses congénères, et constituer des masses de plusieurs dizaines de milliers d'individus par mètre carré. C'est ainsi que d'énormes bouées des US Coast Guards, qui balisent les voies de navigation dans les Grands Lacs, ont coulé sous le poids des coquillages agglutinés autour des chaînes d'ancrage et sur les bouées ellesmêmes.

En quelques années, malgré des dispositions réglementaires prises dès 1989 aux ÉtatsUnis et au Canada pour enrayer son extension et les campagnes d'information du public, la moule zébrée a envahi les Grands Lacs, puis le Saint-Laurent (CLAUDI et MACKIE, 1994), l'Hudson et l'immense bassin du Mississippi, de Saint-Paul à la Nouvelle-Orléans, comme l'indique sa distribution entre 1988 et janvier 1995 (figure 2).

En 1995, la moule zébrée est installée dans 62 lacs nord-américains. Les densités fluctuent d'une année à l'autre : on observe ainsi en 1995 une baisse dans l'lllinois, attribuée à une mauvaise qualité d'eau et une faible fixation en Louisiane en relation avec les fortes températures de l'été 1995 (BENSON et BOYDSTUN, 1995).

\subsection{La mobilisation contre l'envahisseur}

Face à la menace identifiée dès 1989 , la réaction sociale a pris trois directions : utilisation de l'arme réglementaire; initiation de programmes de recherche; large information des scientifiques, des industriels usagers des eaux douces, et du public.

Aux États-Unis comme au Canada, des dispositions réglementaires ont été prises pour limiter l'extension de la moule zébrée dans les eaux intérieures. Le Congrès des États-Unis a voté, en 1990, la première loi visant à éviter et à contrôler l'infestation des eaux intérieures par la moule zébrée et d'autres espèces aquatiques nuisibles : le “ Non Indigenous Species Control Act " (Public Law 101-646 Nov. 29, 1990).

Cette loi définit les espèces aquatiques nuisibles en ces termes : "une espèce non indigène qui menace la diversité ou l'abondance d'espèces indigènes ou la stabilité écologique des eaux infestées ou les activités commerciales, agricoles, aquacoles ou récréatives dépendant de ces eaux."

Elle crée un organisme de coordination des actions : The Aquatic Nuisance Special Task Force (ANS), dont dépend un "Great Lake Panel ". L'ANS dispose d'un financement public et privé ; sa mission consiste à proposer des moyens réglementaires, encourager la coopération avec le Canada et diffuser l'information.

Aux États-Unis, une réglementation a été édictée en application du Non Indigenous Species Control Act. Elle est applicable depuis le 10 mai 1993 et impose de maintenir une salinité d'au moins $30 \mathrm{~g} / \mathrm{l}$ dans l'eau de ballast des bateaux entrant dans les Grands Lacs. Le texte admet cependant des dérogations à cette obligation, comme la rétention de l'eau de ballast durant tout le voyage du bateau dans les Grands Lacs.

A défaut d'une réglementation toujours longue à promulguer, des recommandations ont été proposées au Canada dès 1989, en vue d'éviter des importations d'organismes avec l'eau douce des ballasts. Elles figurent dans le "Voluntary guidelines for the Control of Ballast Water Discharges From Ships Proceeding to the St Lawrence River to Great Lakes ". II est recommandé dans ce document de remplacer l'eau douce des ballasts par de l'eau de mer avant d'entrer dans les eaux côtières nord-américaines (WILEY, 1995).

Sur le plan de la recherche, tant aux États-Unis qu'au Canada, la puissance publique finance des travaux universitaires à caractère fondamental sur la biologie des moules zébrées et les méthodes de lutte les mieux appropriées. Le principal programme fait partie du Sea Grant Program, créé aux États-Unis en 1966 pour mieux connaître et utiliser les Grands Lacs 
et le milieu marin. En quatre ans (1991-1994), le Sea Grant Program a consacré 10,3 millions de dollars à des recherches sur la moule zébrée (SEA GRANT ZEBRA MUSSEL REPORT, 1995).

Des groupements industriels ont, d'autre part, développé des programmes de recherche appliquée ; pour le secteur électrique, c'est l'Edison Power Research Institute (EPRI), aux ÉtatsUnis, et le producteur d'électricité Ontario Hydro, au Canada, qui ont le plus financé de recherches, mais de nombreuses autres initiatives ont été prises.

Il va de soi que les bénéficiaires de cette manne ne sont pas exclusivement des équipes universitaires ; le "ZEB business " concerne aussi, et surtout, les bureaux d'études et fabricants de produits et d'appareillages adaptés à la lutte anti-salissure.

Autour de la moule zébrée, une intense communication scientifique s'est développée. Depuis 1991, se tient un colloque annuel (alternativement aux États-Unis et au Canada) pour faire le point des connaissances acquises dans le domaine de la recherche fondamentale comme dans celui des applications industrielles à la lutte anti-salissure.

Le patronage de ces colloques est assuré par les principaux partenaires des programmes de recherche : EPRI, Environnement Canada, Great Lake Sea Grant Network, Ontario Hydro, US Fish and Wildlife Service...

Depuis 1995, le colloque annuel n'est plus exclusivement consacré à la moule zébrée ; il aborde les autres espèces introduites classées parmi les espèces nuisibles (nuisance organisms). Onze espèces animales et végétales étaient identifiées, en 1992, comme exotiques dans les Grands Lacs. Parmi celles-ci figurent deux espèces de poissons d'origine européenne : la grémille (Gymnocephalus cernuus) et la lamproie marine (Petromyzon marinus). Leur impact sur les populations autochtones pélagiques et benthiques fait actuellement l'objet d'études dans le cadre du programme Sea Grant.

\subsection{L'ampleur des dommages pour l'industrie nord-américaine}

Tous les secteurs industriels utilisant des eaux de surface sont touchés par les nuisances induites par la moule zébrée ; le US National Biological Service prévoit, en 2000, un coût annuel de 5 milliards de dollars pour l'industrie (SEA GRANT ZEBRA MUSSEL REPORT, 1995).

\section{Production d'eau potable}

Deux types de nuisances sont cités par les exploitants d'usines installées sur les Grands Lacs :

- réduction de la capacité de pompage, qui conduit à surdimensionner les prises d'eau, et formation de frazil en hiver dans les canalisations infestées ;

- colmatage des prises d'eau par des algues filamenteuses, dont le développement est favorisé par l'éclaircissement de l'eau résultant de la consommation du phytoplancton par les moules zébrées; il a ainsi fallu enlever en moyenne sur un site $750 \mathrm{~kg}$ d'algues chaque jour, de mai à août.

Les moyens de contrôle adoptés sont des traitements chimiques au chlore (LE PAGE, 1993) ou au permanganate de potassium au niveau des prises d'eau (BALOG et al., 1995).

\section{Centrales thermiques}

Cinquante-trois centrales thermiques sont installées sur les rives des Grands Lacs. Toutes les parties des circuits dans lesquelles l'eau de refroidissement circule à une vitesse inférieure à $1 \mathrm{~m} / \mathrm{s}$ sont colonisées par les moules. Du côté américain, les compagnies utilisent cinq types de traitement anti-salissures (ARMOR et al., 1993), dont les plus fréquents sont le nettoyage mécanique des circuits, la chloration et l'usage de toxiques non oxydants. De nombreuses centrales utilisaient une procédure efficace contre la faune indigène, mais qui s'est 
révélée inadaptée contre les moules zébrées. Des exploitants ont d'abord eu recours au nettoyage manuel par des plongeurs; ils ont ensuite testé des traitements chimiques dans l'urgence, sans qu'une cohérence d'ensemble n'apparaisse.

L'Electric Power Research Institute (EPRI), organisme de recherche pour les producteurs d'électricité américains, a rapidement pris la mesure du problème posé aux exploitants. II a engagé des études et des actions d'information. Un guide sur la surveillance et les méthodes de lutte contre la moule zébrée, destiné aux exploitants de centrales thermiques, a été publié par l'EPRI en décembre 1992 (EPRI, 1992).

Depuis l'apparition de la moule zébrée en 1991 dans le Tennessee, la Tennessee Valley Authority, qui exploite des barrages et des centrales thermiques à flamme et nucleaires, a mis en place une surveillance de la densité de larves planctoniques et des fixations sur tous les sites susceptibles d'être infestés (KERLEY, 1995). Ce programme a débuté en 1992; il a montré que la reproduction des dreissènes restait faible dans les retenues hydroélectriques et sur la plupart des sites de centrales thermiques sur le Tennessee. En revanche, la densité des moules atteint 40000 individus par $\mathrm{m}^{2}$ dans l'Ohio inférieur et une infestation massive a été constatée dans les circuits des centrales installées sur le cours inférieur de l'Ohio (Shawnee Fossil Plant) et sur le Mississippi à Allen Fossil Plant, près de Memphis. Sur ces sites, les moules sont éliminées par un traitement thermique et chimique, déclenché dès que les fixations sont constatées.

Au Canada, la compagnie Ontario Hydro a, dès le début de l'invasion, mis en oeuvre un programme de recherches comportant un volet d'études en laboratoire et de nombreux essais sur des circuits de centrales. Un ouvrage de synthèse a été publié en 1994 (CLAUDI et MACKIE, 1994). A titre provisoire, c'est la chloration, selon un protocole optimisé, qui a été adoptée par Ontario Hydro comme moyen de traitement chimique (EVANS et al., 1992 ; WIANCKO et CLAUDI, 1994).

\section{Centrales hydrauliques}

Les moules se fixent sur les vannes des usines hydrauliques et dans les circuits auxiliaires de refroidissement équipés d'échangeurs à plaques. Hydro Québec est particulièrement concerné par l'infestation des circuits de la centrale de Beauharnais et utilise la chloration à l'instar d'Ontario Hydro, tout en suivant le développement des recherches dans le domaine de la lutte anti-salissure.

\section{La navigation}

Les circuits d'eau brute des bateaux doivent être traités contre les moules zébrées ; le corps des US Coast Guards a joué un rôle moteur dans ce domaine.

\section{Industries diverses}

Raffineries, aciéries, usines chimiques et agro-alimentaires abondent autour des Grands Lacs, dont elles utilisent l'eau brute pour des usages diversifiés. Elles sont évidemment touchées par l'infestation.

\section{LES FACTEURS BIOLOGIQUES ET ÉCOLOGIQUES QUI EXPLIQUENT L'EXTENSION DES MOULES ZÉBRÉES ET LEUR CARACTĖRE INVASIF}

\subsection{Reproduction et croissance}

Le premier facteur qui confère à l'espèce une grande capacité d'extension en milieu lacustre comme dans les grandes rivières est son mode de reproduction.

Le cycle biologique de la moule zébrée est schématisé sur la figure 3 , selon ACKERMAN (1995). II n'y a pas de différence décelable, sur le plan morphologique, entre les stades larvaires des deux espèces présentes en Amérique du Nord. 


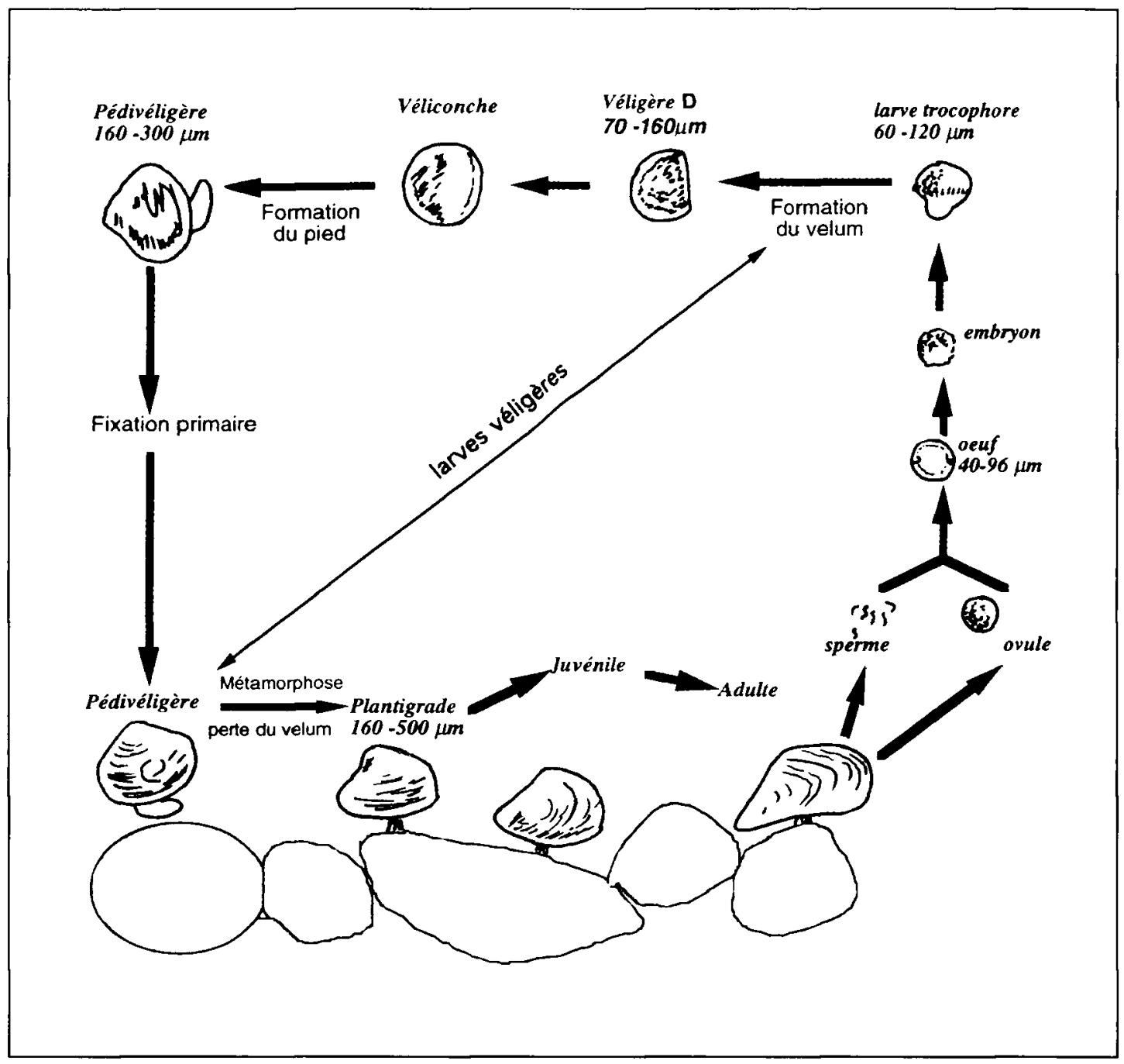

Figure 3

Représentation schématique du cycle biologique de Dreissena polymorpha, adapté d'après ACKERMAN, 1995.

Figure 3

Biological cycle of Dreissena polymorpha, adapted from ACKERMAN, 1995.

Ce cycle peut être schématisé en 3 phases :

- une phase planctonique, qui dure deux à quatre semaines selon la température de l'eau (SPRUNG, 1987 ; BALDWIN, 1995 ; SELTZER-HAMILTON et al., 1995) ;

- une phase de transition, pendant laquelle les larves se fixent et se métamorphosent ;

- une dernière phase benthique, dans laquelle le bivalve est fixé par un byssus comme chez les Mytilidés; la moule zébrée peut toutefois se détacher et possède ainsi une certaine mobilité.

Le second facteur positif est d'ordre trophique : la dreissène présente un large spectre alimentaire ; elle manifeste " un réel opportunisme ", ainsi que le remarque TESTARD (1990). La moule zébrée utilise une grande variété de particules en suspension dans l'eau ; c'est un organodétritiphage, qui consomme aussi bien des algues planctoniques que des détritus 
organobactériens. D'après la revue de TESTARD (1990), la sélectivité des particules est comprise entre $15 \mu \mathrm{m}$ et $45 \mu \mathrm{m}$ avec une sélection maximale centrée autour de 20-25 $\mu \mathrm{m}$, qui correspond à la dimension des taxons phytoplanctoniques généralement majoritaires dans les eaux continentales. Des travaux américains ont par ailleurs révélé que les moules zébrées consomment aussi du microzooplancton jusqu'à des tailles de $100-200 \mu \mathrm{m}$, comme des Véligères de dreissènes, des Rotifères, des petits Cladocères (DEAN et al., 1995).

De par sa grande tolérance thermique, la moule zébrée peut occuper un vaste espace géographique. La dreissène est adaptée aux climats continentaux caractérisés par une grande amplitude thermique : ainsi, elles survivent en hiver dans les eaux glacées et supportent en Moselle pendant plusieurs semaines des températures estivales comprises entre $25^{\circ} \mathrm{C}$ et $28{ }^{\circ} \mathrm{C}$ (MORETEAU et KHALANSKI, 1994). La tolérance thermique des dreissènes en Amérique du Nord leur permet de se développer dans le cours inférieur du Mississippi, dans des eaux dont l'amplitude annuelle va de $10^{\circ} \mathrm{C}$ à $30^{\circ} \mathrm{C}$ (HERNANDEZ et MAC MAHON, 1995). Des animaux acclimatés à $30^{\circ} \mathrm{C}$ meurent en quelques jours à des températures plus élevées : la température létale en 7 jours est de $32^{\circ} \mathrm{C}$, elle est de $31^{\circ} \mathrm{C}$ en 16 jours (MAC MAHON et al., 1994).

Certains facteurs de qualité d'eau sont, en revanche, limitants.

Les moules zébrées ne peuvent pas incorporer le calcium au-dessous d'une concentration de 12 à $14 \mathrm{mg} / \mathrm{l}$, contrairement aux Anodontes dont le seuil est à $3 \mathrm{mg} / \mathrm{l}$ et aux Unios (6-7 mg/l) ; cela explique leur absence de certains lacs en Russie (VINOGRADOV et al., 1995).

Les deux espèces présentes en Amérique du Nord supportent, sans dommage apparent, une salinité de $2 \mathrm{~g} / \mathrm{l}$; la mortalité est significative à partir de $4 \mathrm{~g} / \mathrm{l}$ et la $\mathrm{CL} 50$ se situe vers $6 \mathrm{~g} / \mathrm{l}$ (KENNEDY et al., 1995 ; WRIGHT et al., 1995). D'autres Dreissenidae vivent dans la mer d'Aral à $22 \mathrm{~g} / \mathrm{l}$ (KARATAYEV, 1995).

Un excès de matières en suspension entraîne la production de pseudo-fèces par la moule zébrée, comme c'est le cas chez la moule marine. Des tests en laboratoire ont montré que le bilan énergétique (Scope for Growth) devenait nul à partir d'une teneur en Mest de $80 \mathrm{mg} / \mathrm{l}$ (SCHNEIDER et al., 1995). Avec de l'argile, l'augmentation de la production de pseudo-fèces devient significative à partir de $25 \mathrm{mg} / \mathrm{l}$ (MAC ISAAC et ROCHA, 1995).

Dreissena polymorpha est une espèce oxyphile qui demande, sur le long terme, une oxygénation supérieure à $50 \%$ de saturation; l'optimum étant situé à au moins $80 \%$ de saturation. Dreissena bugensis supporte mieux les basses teneurs en oxygène dissous et pourrait remplacer $D$. polymorpha dans les eaux peu oxygénées (KARATAYEV, 1995). Les moules zébrées peuvent, en revanche, rester fermées à l'air libre pendant plusieurs jours dans une atmosphère humide (TESTARD, 1990).

La vitesse de l'eau peut aussi constituer un facteur limitant pour la croissance de la moule zébrée. La fixation est supprimée quand la vitesse de l'eau dans les circuits industriels excède $1,5 \mathrm{~m} / \mathrm{s}$ (JENNER et JANSSEN-MOMMEN, 1993). Dans le circuit de la centrale thermique de Cattenom, en Moselle, la croissance est optimale pour des vitesses comprises entre 0,1 et $0,5 \mathrm{~m} / \mathrm{s}$; par ailleurs, des observations faites dans les circuits des centrales thermiques exploitées par EDF indiquent l'absence de moules zébrées dans les conduites lorsque la vitesse est supérieure à $1 \mathrm{~m} / \mathrm{s}$. On peut donc estimer que la vitesse optimale pour la croissance de Dreissena polymorpha se situe probablement dans la gamme de $0,1 \mathrm{~m} / \mathrm{s}$ à 0,5 $\mathrm{m} / \mathrm{s}$, qui correspond à la gamme des vitesses couramment rencontrées dans les rivières. A partir de $1 \mathrm{~m} / \mathrm{s}$, la croissance est fortement réduite et les moules sont éliminées à $1,5 \mathrm{~m} / \mathrm{s}$. 


\section{Tableau I}

Croissance de la coquille chez diverses populations de Dreissena polymorpha dans des cours d'eau et lacs européens.

Table I

Shell growth in populations of Dreissena polymorpha from European rivers and lakes.

\begin{tabular}{|c|c|c|c|c|c|}
\hline \multirow[b]{2}{*}{ Rivières et lacs } & \multicolumn{4}{|c|}{ Longueur de coquille (mm) } & \multirow[b]{2}{*}{ Référence } \\
\hline & $\begin{array}{l}\text { Fin du 1er } \\
\text { été }(0+)\end{array}$ & $\begin{array}{l}\text { Fin du 2ème } \\
\text { été }(1+)\end{array}$ & $\begin{array}{l}\text { Fin du 3ème } \\
\text { été }(2+)\end{array}$ & $\begin{array}{l}\text { Fin du tème } \\
\text { éte }(3+)\end{array}$ & \\
\hline $\begin{array}{l}\text { La plupart des rivières } \\
\text { et lacs européens }\end{array}$ & $4,0-5,0$ & 10.0 & $20.0-22,0$ & $20,0-28,0$ & $\begin{array}{c}\text { TESTARD P., } \\
1990\end{array}$ \\
\hline Oise 1985 (France) & $5,0-7,0$ & $16,0-17,0$ & & & \\
\hline $\begin{array}{l}\text { Moselle 1991-1993 } \\
\text { (France) }\end{array}$ & $9.5-15,5$ & $22,1-24,4$ & 29,8 & & $\begin{array}{l}\text { MORETEAU J.C., } \\
\text { KHALANSKI M., } \\
1994\end{array}$ \\
\hline Rhône (France) & $8,0-10,0$ & 15,0 & & & $\begin{array}{l}\text { GARRIC J., } \\
\text { MIGEON B. } \\
1983\end{array}$ \\
\hline $\begin{array}{l}\text { Rhin (Allemagne) } \\
\text { Cours supérieur } \\
\text { Cours inférieur }\end{array}$ & $\begin{array}{l}3,0 \\
13,3\end{array}$ & $\begin{array}{l}14,0 \\
24,0\end{array}$ & & & $\begin{array}{l}\text { JANTZ B., } \\
\text { NEUMANN D., } \\
1992\end{array}$ \\
\hline $\begin{array}{l}\text { Deux lacs région de } \\
\text { Cologne (Allemagne) }\end{array}$ & $\begin{array}{c}2,3 \\
13,0\end{array}$ & & & & $\begin{array}{l}\text { SPRUNG M., } \\
1992\end{array}$ \\
\hline
\end{tabular}

La croissance des bivalves est généralement mesurée par l'augmentation de la longueur de la coquille sur un intervalle de temps donné. La taille de coquille des cohortes de Dreissena polymorpha dans les cours d'eau et plans d'eau européens est indiquée dans le tableau I. II y a de grandes variations selon les lieux, qui s'expliquent essentiellement par la disponibilité en nourriture et le régime thermique annuel. Dans une partie des circuits de la centrale thermique de Cattenom, en Moselle, la croissance des moules zébrées est très rapide pendant l'été : la cohorte issue du premier pic de larves fixées atteint la taille de $15 \mathrm{~mm}$ à la fin de l'été. En comparaison avec les autres données disponibles sur des cours d'eau et plans d'eau européens, il apparaît que cette population se situe parmi celles qui ont le plus fort taux de croissance. La croissance de la coquille peut être calculée à l'aide d'un modèle basé sur trois fonctions représentant la réduction du taux de croissance en fonction de la taille, l'effet de la température de l'eau et la ressource trophique exprimée par la concentration en chlorophylle planctonique (MORETEAU et KHALANSKI, 1994). La croissance de coquille de cette population a été simulée sur trois années à partir des mesures de température et de chlorophylle-a réalisées en Moselle (figure 4). Ce modèle montre la variabilité des tailles atteintes à la fin de la période estivale de croissance, selon les cohortes: la première cohorte provenant des larves fixées en juin atteint une taille de $14-15 \mathrm{~mm}$, alors que la cohorte provenant des fixations du mois d'août n'atteint que 8 à $10 \mathrm{~mm}$.

\subsection{Impact écologique}

L'impact écologique des moules zébrées a fait l'objet de nombreuses études aux États-Unis, financées par le Sea Grant Program. L'extraordinaire développement des moules zébrées au cours des cinq premières années suivant leur découverte dans le lac Saint-Clair s'est accompagné de modifications majeures portant sur la structure biologique et sur le fonctionnement des hydrosystèmes, par le biais d'un impact sur la production planctonique et benthique. Nous allons, dans cette partie, donner un aperçu de la masse imposante de travaux réalisés sur ce thème. 


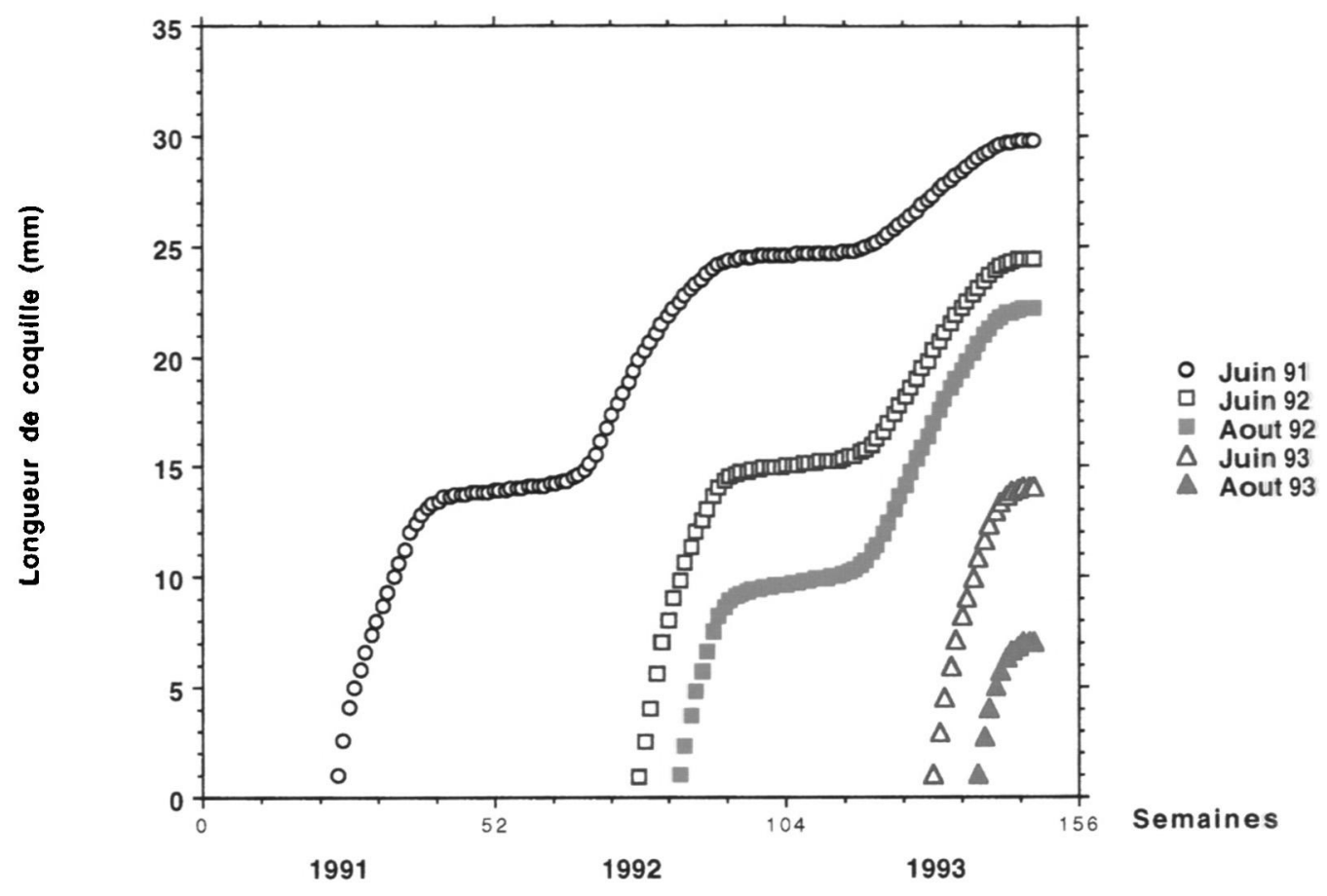

Figure 4

Modélisation de la croissance de coquille pour 5 cohortes de Dreissena polymorpha dans les circuits de la centrale de Cattenom.

\section{Figure 4}

Shell growth modelling of the $\mathbf{5}$ cohorts of Dreissena polymorpha recorded into the Cattenom cooling circuits.

\section{Exclusion compétitive}

Comme toutes les espèces introduites dans un espace naturel, les moules zébrées entrent en compétition avec des espèces indigènes pour l'occupation de l'espace et l'utilisation des ressources nutritives.

La fixation des moules zébrées sur la coquille des bivalves Unionidés indigènes a été remarquée par tous les observateurs. II semble que ces observations ne soient pas anecdotiques, puisque les populations d'Unionidés du lac Saint-Clair et du Lac Érié auraient pratiquement disparu en 1992 (SCHLOESSER, 1995). La fixation des dreissènes sur les coquilles d'Unionidés a aussi été rapportée dans des plans d'eau en Russie (BURLAKOVA, 1995), et nous l'avons fréquemment observée en Moselle. L'impact sur les Unionidés dépend toutefois de la richesse trophique : les Unionidés se maintiennent dans les eaux les plus riches en plancton (HORNBACH et al., 1995). Les dreissènes se fixent également sur la coquille des Gastéropodes Prosobranches indigènes (GREENWOOD et al., 1995). Des fixations, sans doute plus rares, ont aussi été rapportées sur des gammares, des écrevisses, et même sur le ver Turbellarié Dugesia tigrina (CONN, 1993).

Une étude montre que trois espèces d'éponges (Eunapius fragilis, Ephydatia mulleri et Spongilla lacustris) se fixent sur les coquilles de dreissènes et peuvent se développer de manière à " étouffer " la moule en obstruant son siphon inhalant. Les auteurs de cette étude concluent, cependant, que les éponges ne peuvent pas réduire significativement le développement des moules zébrées dans les Grands Lacs (RICCIARDI et al., 1995). Les éponges Spongillidae inhiberaient la fixation de moules zébrées (MINER et al., 1995); cela rejoint les observations faites dans les galeries de la centrale nucléaire de Cattenom en Moselle, qui montrent que les moules zébrées sont absentes des plaques de spongiaires. 
Dans cette ligne, l'effet de l'introduction depuis 1987 de l'Amphipode Corophium curvispinum dans le Rhin inférieur sur le peuplement de moules zébrées doit être cité. Cette petite espèce, qui atteint une densité maximale de 160000 individus par $\mathrm{m}^{2}$, construit des tubes en fixant d'énormes quantités de matières en suspension (197 à $658 \mathrm{~g}$ de poids sec par $\mathrm{m}^{2}$ ). La chute d'abondance des dreissènes dans le Rhin à partir de 1993, de quelques milliers par $\mathrm{m}^{2}$ à quelques dizaines d'individus par $\mathrm{m}^{2}$, a été attribuée à l'occupation des substrats durs par Corophium curvispinum (PAFFEN et al., 1995).

\section{Prédateurs}

La moule zébrée possède de nombreux prédateurs en Europe, comme l'indique la revue faite par TESTARD (1990):

- poissons : le gardon peut consommer préférentiellement des dreissènes juvéniles ; d'autres Cyprinidés comme la brème, la carpe, le chevesne sont des consommateurs occasionnels ou réguliers;

- certains oiseaux aquatiques, comme la Foulque macroule et surtout les Fuligules morillon et miloin, consomment régulièrement des dreissènes; ils peuvent réguler les populations de moules dans certains plans d'eau;

- le rat surmulot (Rattus norvegicus) est aussi un consommateur de moules zébrées.

Comme en Europe, de nombreux prédateurs consomment les moules zébrées en Amérique du Nord:

- des canards plongeurs (TESSIER, 1995);

- des poissons, notamment le " Ruffe " qui n'est autre que la Grémille Gymnocephalus cernuus, espèce européenne introduite dans les Grands Lacs, et Aplodinotus grunnius, le "tambour aquatique " dont la croissance n'est toutefois pas optimale lorsqu'il consomme des dreissènes (FRENCH et BUR, 1995);

- des écrevisses, du genre Orconectes, peuvent être très abondantes en lacs (1-56/m²) et en rivières (3-21/ $\mathrm{m}^{2}$ ), et ainsi contrôler les dreissènes (PERRY et al., 1995). Une expérience en mésocosme a cependant infirmé ce fait (ROEHRS et al., 1995).

\section{Rôle des moules zébrées dans le transfert biologique des polluants}

Plusieurs travaux concluent à un risque de contamination de la chaîne alimentaire aquatique par cette nouvelle source de nourriture, dans laquelle s'accumulent les polluants organiques lipophiles comme des PCB (PILOTE et al., 1995) et des PAH (ROPER et al., 1995). La moule zébrée est d'ailleurs couramment utilisée en Europe comme bioindicateur de pollutions métalliques et radioactives. II faut, à ce sujet, signaler les travaux réalisés à I'Université de Metz (MERSCH et al., 1992 ; GIAMBERINI et PIHAN, 1995).

Par ailleurs, les moules zébrées enrichissent les sédiments en cuivre, zinc et cadmium par l'apport de fèces et pseudo-fèces très enrichies en métaux (KLERKS et al., 1995).

\section{Impact sur la production biologique et les nutriments}

Les investigations menées sur les zones de plus forte abondance en dreissènes (le lac Saint-Clair, le lac Érié et la baie Saginaw dans le Lac Huron) ont mis en évidence de nettes modifications imputables aux moules zébrées. II faut signaler que les premières années suivant leur introduction, la densité des moules zébrées dans ces zones a atteint quelques dizaines de milliers d'individus par $\mathrm{m}^{2}$, et jusqu'à 700000 individus par $\mathrm{m}^{2}$ dans le cas des accumulations produites sur plusieurs saisons de croissance. Les modifications rapportées peuvent être ainsi résumées :

- diminution de la biomasse phytoplanctonique : on est passé d'une valeur estivale de $30 \mu \mathrm{g} / \mathrm{l}$ en chlorophylle-a avant l'invasion à $4 \mu \mathrm{g} / \mathrm{l}$ en 1989 et $9 \mu \mathrm{g} / \mathrm{l}$ en 1994 (BEETON, 1995) ; 
- les dreissènes filtrent intensément les diatomées planctoniques et la réduction de biomasse des diatomées atteint $86 \%$ dans le lac Érié, tandis que la concentration en nutriments (phosphore - azote ammoniacal et silice) et la transparence augmentent (BEETON, 1995) ;

- apparition de blooms de la cyanobactérie Microcystis en 1994 et 1995 dans Saginaw Bay, et en 1995 dans la partie occidentale du lac Érié. Ces fleurs d'eau sont attribuées à l'absence de filtration des Microcystis par les moules zébrées (BEETON, 1995);

- augmentation de la transparence de l'eau liée à la régression du phytoplancton, ce qui favorise des algues vertes Zygnematales (LITTERAL et al., 1995) et quatre espèces de macrophytes qui profitent d'un meilleur éclairement : Potamogeton crispus, Vallisneria americana, Ceratophyllum demersum et Myriophyllum spicatum (HOLLAND, 1995) ;

- diminution de la biomasse du zooplancton par limitation trophique ou par prédation des petites espèces (Rotifères et petits Cladocères) par les moules zébrées (DEAN et al., 1995) ;

- diminution de la biomasse macrobenthique. La biomasse de la faune benthique, en poids sec hors coquille dans la partie orientale du lac Érié, est passée de $1,58 \mathrm{~g} / \mathrm{m}^{2}$ à 11,93 $\mathrm{g} / \mathrm{m}^{2}$ entre 1979 et 1993 , du fait de l'apparition des moules zébrées et des Quagga ( $D$. bugensis) (DERMOTT et KEREC, 1995). En dehors des dreissènes, la biomasse a toutefois diminué (1,61 à $1,04 \mathrm{~g} / \mathrm{m}^{2}$ ) ; la plus forte réduction concerne un amphipode (Diporeia), le bivalve Pisidium et des diptères Chironomidés qui subissent une compétition trophique de la part des moules zébrées. La méiofaune s'accroît en revanche d'un facteur 2 à 6 , car elle bénéficie de l'apport nutritif constitué par les pseudo-fèces de moules.

Nous avons, paradoxalement, peu d'informations sur l'évolution des populations de poissons. Une étude sur le recrutement des poissons dans Saginaw Bay (JUDE, 1995) n'a pas montré de différence dans les larves des principales espèces pélagiques en 1993 : l'alose Alosa pseudoharengus, le " rainbow smelt ", un Osmeridé, et la perche jaune Perca flavescens. Toutefois, le rendement de la pêche de la perche jaune au chalut a fortement baissé au cours des dernières années, ce qui laisse supposer que le recrutement est mauvais malgré une abondance de larves normale. La rareté du zooplancton pourrait expliquer le déclin de cette population de poissons.

\section{SALISSURES BIOLOGIQUES INTRODUITES DANS LES EAUX CONTINENTALES FRANÇAISES}

Le tableau II présente les organismes fixés, identifiés dans les circuits de centrales thermiques exploitées par EDF.

Parmi les végétaux qui se développent sur les structures exposées à la lumière, le groupe des cyanobactéries (algues bleues) est le plus important. II constitue en effet les " algues de coques " qui recouvrent la paroi interne des tours, sur tous les sites équipés d'aéroréfrigérants (APROSI et CHAUVEL, 1990). Des algues vertes filamenteuses sont aussi observées dans les bassins froids d'aéroréfrigérants.

II faut, d'autre part, signaler la grande abondance de végétaux aquatiques dérivant dans les rivières en période automnale. Ils sont normalement éliminés par les filtres rotatifs qui protègent les circuits, mais une brèche sur le filtre peut entraîner le colmatage des échangeurs thermiques placés sur des circuits auxiliaires.

Les animaux présentant la plus grande abondance dans les circuits sont des filtreurs de fines particules, dont le développement est favorisé par l'abondance du phytoplancton dans nos rivières. Ils appartiennent aux groupes suivants :

- spongiaires : formes encroûtantes ou formes buissonnantes fixées sur les parois des conduites, sur tous les sites; 


\section{Tableau II}

Salissures biologiques identifiées en 1995 dans des circuits de centrales thermiques exploitées par EDF. Les taxons les plus abondants sont notés en caractères gras.

(1) Canalisations et bassins à vitesse inférieure à $1 \mathrm{~m} / \mathrm{s}$.

\section{Table II}

Biofouling identified in 1995 in the cooling circuits of power stations operated by EDF. The most abundant taxa are in bold types.

(1) pipes and basins where the water velocity is lower than $1 \mathrm{~m} / \mathrm{s}$.

\begin{tabular}{|c|c|c|c|c|c|c|c|}
\hline Rmieres & Centrales & $\begin{array}{c}\text { Dans les } \\
\text { canalisations }\langle 1\rangle\end{array}$ & $\begin{array}{l}\text { Sur gnilles et fithres } \\
\text { a ta prise d'eau }\end{array}$ & $\begin{array}{c}\text { Condenseurs (echangeurs } \\
\text { themroues tubulaires) }\end{array}$ & $\begin{array}{c}\text { Echangeurs thermiques } \\
\text { à plaques }\end{array}$ & $\begin{array}{l}\text { Cans les aéro } \\
\text { réfrigerants }\end{array}$ & $\begin{array}{l}\text { Espéces invasives } \\
\text { recemment introduttes }\end{array}$ \\
\hline Seine & $\begin{array}{c}\text { NogenUSeine } \\
\text { Montereau } \\
\text { Vilry/Seine }\end{array}$ & $\begin{array}{c}\text { Bythinia sp. } \\
\text { Sphaerium sp. } \\
\text { Anodonte sp. } \\
\text { Physa sp. } \\
\text { Dreissena polymorpha } \\
\text { Fredencella sultana } \\
\text { Paludicella } \\
\text { Dreissena polymorpha } \\
\text { Dreissena polymorpha }\end{array}$ & & & $\begin{array}{l}\text { Bythinia sp. } \\
\text { Spharerium sp. } \\
\text { Anodonta sp. }\end{array}$ & Algues de coque & \\
\hline Rhin & Fessenheim & & $\begin{array}{c}\text { Végétaux dérivants: } \\
\text { Potarmogoton pectinatus }\end{array}$ & & & & \\
\hline & La Maxe & Dreissens polymorpha & & & & & \\
\hline Moselle & Cattenom & \begin{tabular}{|c|} 
Dreissena polymorpha \\
Cordylophora sp \\
Bythinia sp. \\
Viviparus sp. \\
Unionides \\
Spongiaires \\
\end{tabular} & $\begin{array}{l}\text { Végétaux derivants: } \\
\text { Mynophyilum spicatum }\end{array}$ & & & Algues de coque & $\begin{array}{c}\text { Conticula sp } \\
\text { Corophium curvispinum } \\
\text { depuis } 1994\end{array}$ \\
\hline Meuse & Chooz & $\begin{array}{l}\text { Dreissena polymorpha } \\
\text { Plumatella fungosa } \\
\text { Plumstella omarginata }\end{array}$ & & & & $\begin{array}{c}\text { Plumatellia hungosa } \\
\text { Plumatelle emarginara }\end{array}$ & $\begin{array}{c}\text { Corophium curvispinum } \\
\text { depuis } 1994\end{array}$ \\
\hline \multirow{2}{*}{ Loure } & Dampierre & Plumatella fungosa & & & & $\begin{array}{c}\text { Plumbtolla fungosa } \\
\text { Fredenicelfe sultana } \\
\text { Alguas do coque } \\
\text { Cladophore sp. }\end{array}$ & \\
\hline & Chinon & Plumatella fungosa & & & $\begin{array}{l}\text { Piumatella fungosa } \\
\text { Gasteropodes }\end{array}$ & $\begin{array}{l}\text { Piumatella tungosa } \\
\text { Algues de coque }\end{array}$ & \\
\hline \multirow[b]{2}{*}{ Rhóne } & Bugey & Dreissena polymorpha & & & & Cladophora sp. & $\begin{array}{l}\text { Corbicula sp } \\
\text { depuis } 1990\end{array}$ \\
\hline & Cruas & Dreissena polymorpha & & & & $\begin{array}{l}\text { Cladophora Sp. } \\
\text { Algues de coque } \\
\text { Dopots minéraux }\end{array}$ & \\
\hline Garonne & Golfech & $\begin{array}{c}\text { Corbiculas sp. } \\
\text { Oreissena polymorpha } \\
\text { Limnea sp. } \\
\text { Anadonta sp. } \\
\text { Spongilla lacustris }\end{array}$ & Cladophora sp & Corbicula sp. & $\begin{array}{c}\text { Dreissena polymorpha } \\
\text { Corbicwla sp. } \\
\text { Limnea sp } \\
\end{array}$ & $\begin{array}{c}\text { Corbicula sp } \\
\text { Limnea sp. } \\
\text { Anadonta sp } \\
\text { Oreissena polymorpha } \\
\text { Cladophora }\end{array}$ & $\begin{array}{l}\text { Corbicula sp. } \\
\text { depuis } 1989\end{array}$ \\
\hline
\end{tabular}

- hydraires : Cordylophora caspia se présente sous l'aspect de petits tubes formant un gazon sur toutes les parois ; très abondants en Moselle à Cattenom ;

- bryozoaires : colonies massives de Plumatella fungosa et d'autres espèces présentes dans les conduites immergées et dans les bassins froids des aéroréfrigérants, particulièrement abondants dans les circuits des centrales en Loire : Dampierre-en-Burly et Chinon (APROSI et al., 1988) ;

- moule zébrée : Dreissena polymorpha se trouve fixée sur les parois des canalisations et bassins ; l'espèce est abondante en Moselle, dans le Rhône et dans le bassin de la Seine. A Cattenom, en 1989, $400 \mathrm{~m}^{3}$ de coquilles et de vase ont été retirés des conduites, en amont des pompes alimentant les circuits auxiliaires classiques et nucléaires. La biologie de la moule zébrée sur le site de Cattenom a été étudiée par l'Université de Metz. On connaît ainsi les périodes de fixation des larves et le taux de croissance des organismes fixés. A partir de ces connaissances, un modèle mathématique a été construit par EDF-DER et l'Université de Metz. Il permet de calculer la croissance de chaque cohorte en fonction de la température et de la teneur de l'eau en chlorophylle planctonique.

Bien que la moule zébrée soit peu abondante dans la Garonne, elle a envahi les circuits de la centrale de Golfech, où des quantités comparables à celles de Cattenom ont été retirées en 1995. 


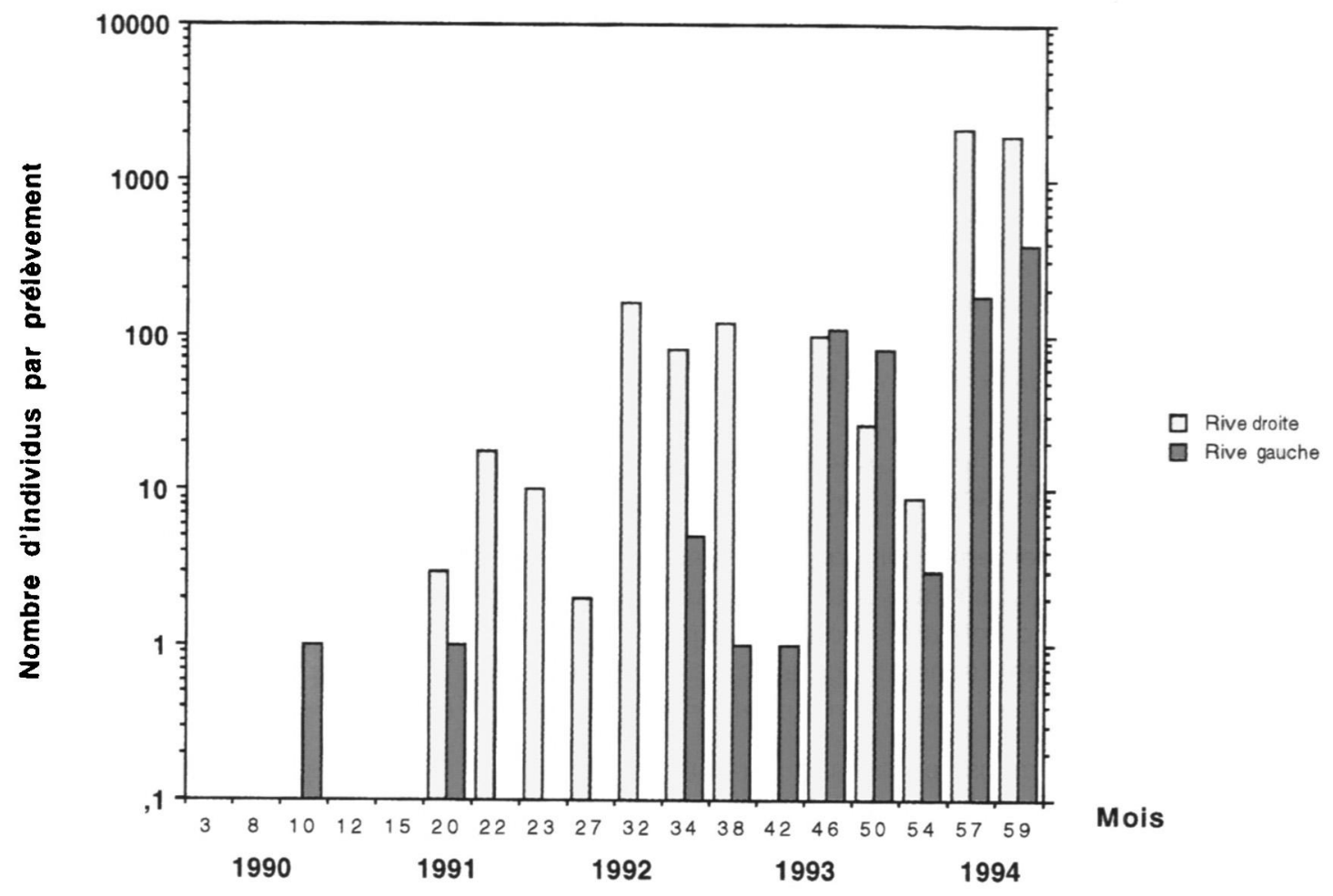

Figure 5

Abondance de Corbicula sp. dans le Rhône en aval de la centrale du Bugey, d'après les relevés du CEMAGREF.

Figure 5

Abundance of Corbicula sp. in the Rhône River downstream to Le Bugey power station, from the CEMAGREF macroinvertebrates monitoring.

Des Gastéropodes (Bythinia, Viviparus, Limnea...) et d'autres bivalves (Sphaerium, Unio, Anodonta) sont également présents dans les circuits et se retrouvent dans des échangeurs thermiques.

Deux espèces, récemment introduites dans nos cours d'eau, constituent une menace pour les circuits d'eau de refroidissement. Leur extension sera suivie, dans les années à venir, à partir des observations et expertises réalisées sur les circuits des centrales thermiques exploitées par EDF.

- le Clam asiatique : Corbicula fluminea, est un bivalve présent dans certains cours d'eau européens (rivières du sud-ouest de la France, Rhin aux Pays-Bas), qui vit normalement dans le sédiment. II abonde dans les circuits de distribution d'eau d'irrigation du bassin de la Garonne (DUBOIS, 1995) et est présent dans les bassins froids des aéroréfrigérants à la centrale de Golfech. La quantité de Corbicula retirée d'un bassin froid, de mai à fin septembre 1990, a été estimée à environ $10 \mathrm{~m}^{3}$. Les coquillages se trouvaient dans les zones les moins turbulentes du bassin et sous les tulipes d'arrivée d'eau. La présence de Corbicula en Moselle a été signalée en 1994 (BACHMANN et al., 1995) et il est actuellement présent dans le Haut Rhône, en amont de Lyon, depuis 1990 (figure 5), d'après les relevés de macroinvertébrés effectués en aval de la centrale du Bugey (CEMAGREF, 1990 à 1994).

- le crustacé Amphipode Corophium curvispinum, présent dans le Rhin, n'a été signalé fin 1995 que dans deux rivières du nord-est de la France : la Meuse à la centrale de Chooz, et la Moselle (DUVIVIER et VANMAELE, 1995 ; BACHMANN et al., 1995). Cette petite espèce tubicole, qui peut prendre un développement considérable, pourrait être un compétiteur de la moule zébrée pour l'espace. 


\section{MOYENS DE LUTTE CONTRE LES SALISSURES BIOLOGIQUES UTILISÉS DANS LES CENTRALES THERMIQUES EXPLOITÉES PAR EDF}

Contre le voile bactérien qui se développe sur les tubes de condenseurs, toutes les centrales d'EDF installées sur rivières sont équipées d'un dispositif de nettoyage automatique par boules en mousse.

En règle générale, les circuits des centrales sont nettoyés hors d'eau, une fois par an, lors des arrêts de tranches. II s'agit d'un nettoyage mécanique pratiqué par décollement des organismes fixés, avec des jets d'eau sous pression ou par raclage des parois. Lorsque des circuits communs à deux tranches restent en eau, le nettoyage est effectué manuellement par des plongeurs, comme c'est le cas à Cattenom ; les premières années, lorsque les quantités de moules étaient importantes, le chantier durait environ 4 mois par an sur chaque ouvrage correspondant à deux tranches.

Les seuls traitements chimiques utilisés dans les centrales thermiques exploitées par EDF sont le nettoyage chimique des échangeurs thermiques à plaques et la chloration. II s'agit, dans le premier cas, d'enlever les dépôts organiques et minéraux sur les surfaces d'échange thermique en pratiquant un nettoyage avec de l'acide oxalique, des dispersants et de la soude. Ce type de traitement en circuit fermé n'engendre pas de rejet en rivière.

La chloration massive des aéroréfrigérants consiste à fermer la purge du circuit et à injecter rapidement de l'eau de javel, pour obtenir une concentration en chlore de quelques $\mathrm{mg} / \mathrm{l}$ à quelques dizaines de $\mathrm{mg} / \mathrm{l}$. La purge n'est réouverte qu'après 6 à 7 heures, lorsque la teneur en chlore résiduel devient inférieure à $0,1 \mathrm{mg} / \mathrm{l}$. Ce procédé permet de nettoyer le bassin froid et les structures de ruissellement sur lesquelles peuvent se développer des algues vertes et des cyanobactéries. Ce traitement est actuellement peu pratiqué (une à deux fois par an sur un site, en 1994-1995). II constitue cependant un moyen disponible pour les exploitants en cas d'encrassement biologique important. La chloration continue à faible dose est pratiquée sur les circuits des centrales en bord de mer exploitées par EDF ; ce type de traitement est aussi utilisé en Amérique du Nord et en Europe pour contrôler le développement des salissures biologiques.

C'est en équipant les circuits de filtres rotatifs ou de grilles amovibles aisément nettoyables au niveau du retour au condenseur que le risque de colmatage des échangeurs thermiques posé par les algues de coques est actuellement maîtrisé.

Des filtres rotatifs ou des grilles amovibles permettent d'éliminer le risque d'entraînement de Bryozoaires qui se développent dans les bassins froids des aéroréfrigérants des centrales d'EDF. Dans les canalisations et bassins des ouvrages d'amenée-reprise, le nettoyage des parois est pratiqué. Le maintien d'un courant d'eau avec des vitesses suffisamment élevées permet, en revanche, de réduire le développement des Bryozoaires (APROSI et al., 1988); ce fait a conduit EDF DER à recommander des permutations de pompes, de manière à éviter de laisser pendant de longues durées des parties de circuits en eau peu courante.

Des investigations ont été engagées à partir de 1990 par Electricité de France en vue d'assurer la protection des circuits auxiliaires de la centrale de Cattenom contre les moules zébrées. L'installation de filtres à débris en amont des échangeurs des circuits auxiliaires permet de réduire l'encrassement de ces échangeurs; ces dispositifs n'évitent toutefois pas le nettoyage des conduites en eau.

L'introduction de nouvelles espèces invasives ou le développement excessif d'espèces indigènes peut, dans l'avenir, rompre l'équilibre assuré par ces procédés mécaniques; il faudrait alors recourir à d'autres traitements sélectionnés sur quatre critères : efficacité, absence de rejets toxiques rémanents, simplicité de mise en oeuvre du procédé et coût supportable. C'est dans cette perspective que des traitements thermiques et chimiques permettant d'éliminer les animaux fixés sont étudiés (KHALANSKI, 1993). 


\section{BIBLIOGRAPHIE}

ACKERMAN J.D., 1995. Zebra mussel life history. In Proceedings of the Fifth International Zebra Mussel and Other Aquatic Nuisance Organisms Conference, Toronto, Ontario, 1-8.

APROSI G., BIDARD F., NEPVEU DE VILLEMARCEAU C., 1988. Expérience d'exploitation des aéroréfrigérants des centrales nucléaires françaises: hydrobiologie-chimie aquatique. Revue Générale Nucléaire, 5, 425-429.

APROSI G., CHAUVEL D., 1990. The quantity of algae settling on the inside shell of cooling towers and the consequences on the wear of the shell. 7ème Congrès AIRH, Léningrad. Rapport EDF/DER HE/31-90.05, 9 p.

ARMOR A.F., TSOU J.L., WIANKO P.M., 1993. Zebra mussels : the industrial impact. In Proceedings of the Third International Zebra Mussel Conference, Toronto, Ontario, 2-25 2-34, EPRI Report TR-102077, K-1 K-19.

BACHMANN V., CIEGELKA E., WAGNER P., USSEGLIO-POLATERA P., MORETEAU J.C., 1995. Establishment of the Amphipod Corophium curvispinum and the Asiatic Clam Corbicula sp., in the French part of the Mosel river. Hydroécologie Appliquée, 7, 1-2.

BALDWIN B.S., 1995. Settlement and metamorphosis of larval Zebra and Quagga Mussels: implications for their colonization and spread. In Fifth International Zebra Mussel and Other Aquatic Nuisance Organisms Conference, Toronto, Ontario.

BALOG G.G., NEIMEYER T.F., DAVIS L.S., SOKHEY A., SCOTT D.E., CUSTODIO O., 1995. Baltimore City adopts a proactive approach to Zebra mussel control using potassium permanganate. In Proceedings of the Fifth International Zebra Mussel and Other Aquatic Nuisance Organisms Conference, Toronto, Ontario, 9-21.

BEETON A.M., 1995. Ecosystem impacts of the Zebra Mussel Dreissena polymorpha. Fifth International Zebra Mussel and Other Aquatic Nuisance Organisms Conference, Toronto, Ontario.

BENSON A.J., BOYDSTUN C.P., 1995. Zebra Mussel distribution in the United States. Zebra Mussel Workshop 95. US army Corps of Engineers, New Orleans.

BURLAKOVA L., 1995. The relationship between Dreissena polymorpha and other benthic animals. Fifth International Zebra Mussel and Other Aquatic Nuisance Organisms Conference, Toronto, Ontario.

CEMAGREF, 1990 à 1994. Suivi hydrobiologique du Rhône au Bugey. In Rapports annuels d'environnement du CNPE du Bugey pour les années 1989, 1990, 1991, 1992, 1993, 1994.

CLAUDI R., MACKIE G.L., 1994. Practical Manual for Zebra Mussel Monitoring and Control. Lewis Publishers, $227 \mathrm{p}$.

CONN D.B., 1993. Parasitism and other associations between Dreissenid mussels and native animals in the St Lawrence River. Proceedings of the Third International Zebra Mussel and Other Aquatic Nuisance Organisms Conference, Toronto, Ontario. EPRI Report TR-102077, 2-25 2-34.

DEAN D.M., VANNI M.J., AUCH J.D., HEADWORTH J.L., LAYNE A.F., LAYNE C.D., 1995. Impacts of Zebra Mussels on phyto and zooplankton community structure in Western Lake Erie. In Fifth International Zebra Mussel and Other Aquatic Nuisance Organisms Conference, Toronto, Ontario.

DERMOTT R., KEREC D., 1995. Changes in the deep-water benthos of Eastern Lake Erie between 1979 and 1993. In Proceedings of the Fifth International Zebra Mussel and Other Aquatic Nuisance Organisms Conference, Toronto, Ontario, 57-64.

DUBOIS C., 1995. Corbicula fluminea : un mollusque opportuniste. Agence de l'Eau Adour-Garonne ETE 95, 1-16. 
DUVIVIER L., VANMAELE R., 1995. Centrale de Chooz B. Biologie. Rapport de synthèse. Rapport LABORELEC C03-800-95-005/F/LDU/RVM, 18 p.

EPRI, 1992. Zebra mussel monitoring and control guide. EPRI Report TR-101782.

EVANS D.W., GRIFFIFTHS J.J., KOOPMANS R., KOWALEWSKI E.G., PATRICK P.H., SPENCER F.S., 1992. Options for controlling Zebra Mussels. Ontario Hydro Research Review, 7, 1-25.

FRENCH J.R.P., BUR M.T., 1995. Impact of Zebra Mussels on growth of freshwater drum in Western lake Erie. In Fifth International Zebra Mussel and Other Aquatic Nuisance Organisms Conference, Toronto, Ontario.

GARRIC J., MIGEON B., 1983. Etude du cycle de reproduction de Dreissena polymorpha sur le Rhône en aval de Lyon. Rapport EDF-DER, HE/31-83.22, 42 p.

GIAMBERINI L., PIHAN J.C., 1995. Tissular and cellular localization of lead in the freshwater Mussel (Dreissena polymorpha). The role of hemocytes and excretory organs in transport and detoxification mechanisms. Fifth International Zebra Mussel and Other Aquatic Nuisance Organisms Conference, Toronto, Ontario.

GREENWOOD K.S., ALEXANDER J.E., THORP J.H., 1995. Selective infestation of Dreissena polymorpha on large Prosobranch snails in the Ohio River. Fifth International Zebra Mussel and Other Aquatic Nuisance Organisms Conference, Toronto, Ontario.

HERNANDEZ M.R., MAC MAHON R.F., 1995. Investigation of geographical variation on the thermal tolerance of Zebra mussels, Dreissena polymorpha. Fifth International Zebra Mussel and Other Aquatic Nuisance Organisms Conference, Toronto, Ontario.

HOLLAND R.E., 1995. Increased abundance of epiphytic diatoms in post-Zebra Mussel Hatchery Bay, Western lake Erie. Fifth International Zebra Mussel and Other Aquatic Nuisance Organisms Conference, Toronto, Ontario.

HORNBACH D.J., BAKER S.H., DENEKA T., 1995. What are the physiological responses of Unionid Mussels to colonization by Zebra Mussels? Fifth International Zebra Mussel and Other Aquatic Nuisance Organisms Conference, Toronto, Ontario.

JANTZ B., NEUMANN D., 1992. Shell growth and aspects of the population of Dreissena polymorpha in the Rhine River. In The zebra mussel Dreissena polymorpha, Neumann D. and Jenner H.A. (eds.), Limnologie Aktuell, 4, 49-66.

JENNER H.A., JANSSEN MOMMEN J.P.M., 1993. Monitoring and control of Dreissena polymorpha and other macrofouling bivalves in the Netherlands. In Zebra Mussels Biology, Impact and Control, Nalepa T.F. and Schloesser D.W. (eds.), Lewis Publishers, 537-554.

JUDE J., 1995. The influence of Zebra Mussels on the recruitment of Saginaw Bay Fishes. In Sea Grant Zebra Mussel Report. An update of Research and Outreach, 1988-1994. Ohio State University, 11.

KARATAYEV A., 1995. Factor determining the distribution and abundance of Dreissena polymorpha in lakes, dam reservoirs and channels. Proceedings of the Fifth International Zebra Mussel and Other Aquatic Nuisance Organisms Conference, Toronto, Ontario, 227-234.

KENNEDY V., MAC ININCH S., WRIGHT D., SELTZER-HAMILTON E., 1995. Salinity and Zebra and Quagga mussels. Fifth International Zebra Mussel and Other Aquatic Nuisance Organisms Conference, Toronto, Ontario.

KERLEY B.L., 1995. Tennessee Valley Authority and the Zebra Mussel distribution, Monitoring and control. Zebra Mussel Workshop 95. US army Corps of Engineers, New Orleans.

KHALANSKI M., 1993. Testing of five methods for the control of Zebra Mussels in cooling circuits of power plants located on the Moselle River. Third International Zebra Mussel Conference. EPRI Report TR-102077. Notes internes de la Direction des Etudes et Recherches 94NV0003, $29 \mathrm{p}$. 
KINZELBACH R., 1992. The main features of the phylogeny and dispersal of the Zebra mussel Dreissena polymorpha. In The zebra mussel Dreissena polymorpha, Neumann D. and Jenner H.A. (eds.), Limnologie Aktuell, 4, 6-17.

KLERKS P., FRALEIGH P., LAWNICZAK J., JERRY J., SAVINO J., 1995. Impact of Zebra Mussel (Dreissena polymorpha) on the environmental cycling of metals. Fifth International Zebra Mussel and Other Aquatic Nuisance Organisms Conference, Toronto, Ontario.

LÉGLIZE L., OLLIVIER M., 1981. Mise au point bibliographique sur la biologie et l'écologie de Dreissena polymorpha Pallas (1771) : répartition géographique en France et dans les pays limitrophes. EDF/DER Rapport HE/31-81.37.

LE PAGE W.L., 1993. Mussel management at Monroe. Past, present and future. In Proceedings of the Third International Zebra Mussel Conference, Toronto, Ontario. EPRI Report, 1-57 $1-79$.

LITTERAL R.L., PILLSBURY R.W., LOWE R.L., 1995. The response of the benthic algal community of Saginaw Bay, near Charity Islands, to changes in light penetration. In Proceedings of the Fifth International Zebra Mussel and Other Aquatic Nuisance Organisms Conference, Toronto, Ontario, 275-290.

MAC ISAAC H.J., ROCHA R., 1995. Effects of suspended clay on feeding behaviour of Dreissena polymorpha. Fifth International Zebra Mussel and Other Aquatic Nuisance Organisms Conference, Toronto, Ontario.

MAC MAHON R.F., MATTHEWS M.A., USSERY T.A., CHASE R., CLARKE M., 1994. Further studies on Heat tolerance in Zebra Mussels : effect of temperature Acclimatization and chronic exposure to lethal temperature. In Proceedings of the Fourth International Zebra Mussel Conference, Madison, Wisconsin, Wisconsin Sea Grant Institute, 251-272.

MERSCH J., JEANJEAN A., SPOR H., PIHAN J.C., 1992. The freshwater mussel Dreissena polymorpha as a bioindicator of trace metals, organochlorines and radionucleides. In The zebra mussel Dreissena polymorpha, Neumann D. and Jenner H.A. (eds.), Limnologie Aktuell, 4, 227-244.

MINER J.G., STEWART T., SNYDER F.L., KELCH D.O., 1995. Distribution, abundance and growth of freshwater sponges (Spongillidae) in Western lake Erie : projecting impact on Zebra Mussels. Contribution to Fifth International Zebra Mussel and Other Aquatic Nuisance Organisms Conference, Toronto, Ontario.

MORETEAU J.C., KHALANSKI M., 1994. Settling and growth of $D$. polymorpha in the raw water circuits of the Cattenom Nuclear Power Plant (Moselle, France). Contribution to Fourth International Zebra Mussel Conference, Madison, Wisconsin, Wisconsin Sea Grant Institute, 553-574.

MORTON B., 1993. The anatomy of Dreissena polymorpha and the evolution and success of the Heteromyarian form in the Dreissenoidea. In Zebra Mussels Biology, Impact and Control, Nalepa T.F. and Schloesser D.W. (eds.), Lewis Publishers, 185-215.

PAFFEN B.G.P., VAN DER VELDE G., BIJ DE VAATE A., JENNER H.A., 1995. Development of the Amphipod Corophium curvispinum and its effects on the lithophilic macroinvertebrates in the Dutch Lower Rhine. Fifth International Zebra Mussel and Other Aquatic Nuisance Organisms Conference, Toronto, Ontario.

PERRY W.L., LODGE D.M., LAMBERTI G.A., 1995. Crayfish predation on Zebra Mussels in Midwestern streams. Fifth International Zebra Mussel and Other Aquatic Nuisance Organisms Conference, Toronto, Ontario.

PILOTE M., PLANAS D., SYLVESTRE M., 1995. Who gets more contaminated : Zebra mussels (Dreissena polymorpha) or Unionids? Fifth International Zebra Mussel and Other Aquatic Nuisance Organisms Conference, Toronto, Ontario. 
RICCIARDI A., REISWIG H.M., SNYDER F.L., KELCH D.O., 1995. Lethal overgrowth of Dreissenid mussels by freshwater sponges : potential biological control? Fifth International Zebra Mussel and Other Aquatic Nuisance Organisms Conference, Toronto, Ontario.

ROEHRS J.M., PILLSBURY R.W., LOWE R.L., ROEHRS M.S., FRANCOEUR S.V., 1995. The impact of crayfish on Zebra Mussels and filamentous green algae in Tawas Bay. Fifth International Zebra Mussel and Other Aquatic Nuisance Organisms Conference, Toronto, Ontario.

ROPER J.M., CHERRY D.S., SIMMERS J.W., 1995. Survival and bioaccumulation of toxicants in the Zebra Mussel at Times Beach, New York. Fifth International Zebra Mussel and Other Aquatic Nuisance Organisms Conference, Toronto, Ontario.

SCHLOESSER D.W., 1995. Infestation of Unionid bivalves by the exotic Zebra Mussel Dreissena polymorpha in large freshwater bodies of Europe and North America. In Fifth International Zebra Mussel and Other Aquatic Nuisance Organisms Conference. Toronto, Ontario.

SCHNEIDER D.W., STOECKEL J., SPACKS R.E., MADON S.P., 1995. Ecological energetics of Dreissena polymorpha in turbid rivers as measured using natural seston. Fifth International Zebra Mussel and Other Aquatic Nuisance Organisms Conference, Toronto, Ontario.

SEA GRANT ZEBRA MUSSEL REPORT 1988-1994, 1995. Ohio Sea Grant Research Program, 54 p.

SELTZER-HAMILTON E.M., MAGEE J.A., WRIGHT D.A., KENNEDY V.S., 1995. Laboratory culture of Dreissena polymorpha and Dreissena bugensis from fertilization to F1 juveniles using estuarine algae. Fifth International Zebra Mussel and Other Aquatic Nuisance Organisms Conference, Toronto, Ontario.

SPIDLE A.P., MARSDEN J.E., MAY B., 1994. Identification of the Great Lakes Quagga Mussel as Dreissena bugensis from the Dnieper River, Ukraine, on the basis of allozyme variation. Can. J. Fish. Aquat. Sci., 51, 1485-1489.

SPRUNG M., 1987. Ecological requirements of developing Dreissena polymorpha eggs. Arch. Hydrobiol., suppl. 79, 69-86.

SPRUNG M., 1992. Observations on shell growth and mortality of Dreissena polymorpha in lakes. In The zebra mussel Dreissena polymorpha, Neumann D. and Jenner H.A. (eds.), Limnologie Aktuell, 4, 19-28.

TESSIER C., 1995. Ecotoxicological impacts of a recently introduced species, Dreissena polymorpha, on native Unionid Mussels in the Illinois River. Fifth International Zebra Mussel and Other Aquatic Nuisance Organisms Conference, Toronto, Ontario.

TESTARD P., 1990. Eléments d'écologie du lamellibranche invasif Dreissena polymorpha Pallas. Etude de la dispersion des larves en région parisienne et de leur fixation. Réponses à la désoxygénation de l'eau. Thèse Doctorat Sciences, Université Paris 6, Paris, $354 \mathrm{p}$.

VINOGRADOV G.A., SMIRNOVA N.F., SOKOLOV V.A., BRAZNITSKY A.A., 1995. Influence of chemical composition of the water on the mollusc Dreissena polymorpha. Fifth International Zebra Mussel and Other Aquatic Nuisance Organisms Conference, Toronto, Ontario.

WIANCKO P.M., CLAUDI R., 1994. The Ontario Hydro final strategy for zebra Mussel control. Fourth International Zebra Mussel Conference, Madison, Wisconsin.

WILEY C.J., 1995. Ballast water control. The Canadian approach. In Proceedings of the Fifth International Zebra Mussel and Other Aquatic Nuisance Organisms Conference, Toronto, Ontario, 489-494.

WRIGHT D.A., SELTZER-HAMILTON E.M., MAGEE J.A., KENNEDY V.S., 1995. Development of Dreissena polymorpha and Dreissena bugensis larvae in salt water. Fifth International Zebra Mussel and Other Aquatic Nuisance Organisms Conference, Toronto, Ontario. 